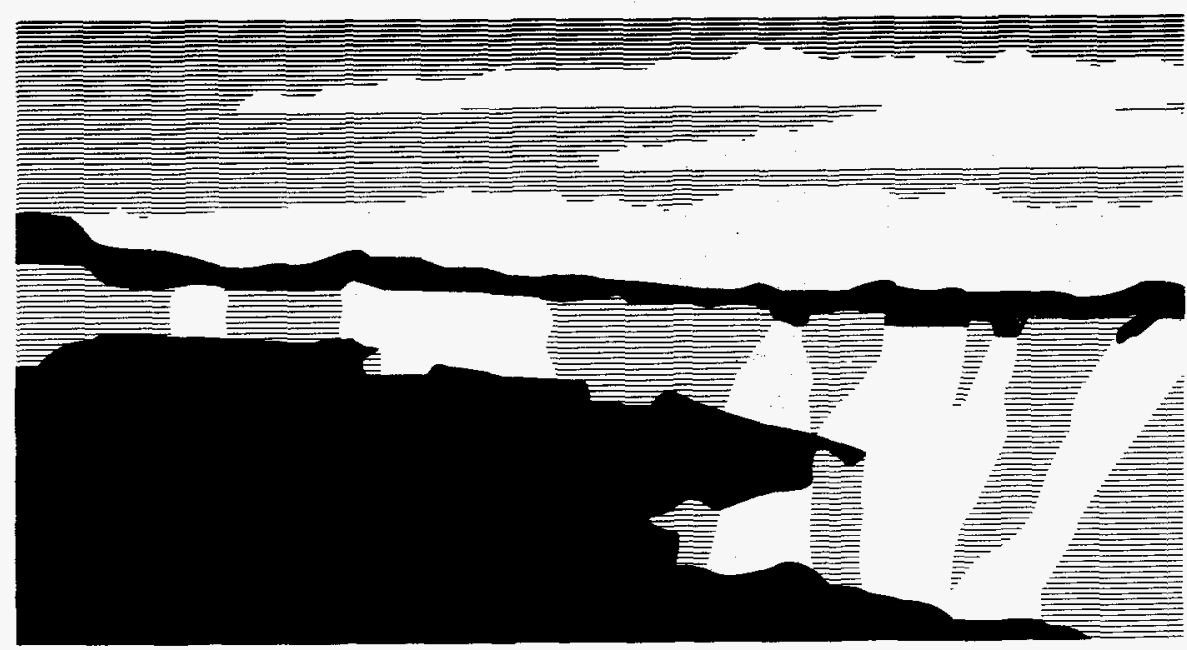

Los Alamos National Laboratory, an affirmative action/equal opportunity empldyer, is operated by the University of California for the U.S. Department of Energy under contract W-7405-ENG-36. By acceptance of this article, the publisher recognizes that the U.S. Government retains a nonexclusive, royalty-free license to publish or reproduce the published form of this contribution, or to allow others to do so, for U.S. Government purposes. The Los Alamos National Laboratory requests that the publisher identify this article as work performed under the auspices of the U.S. Department of Energy. 


\title{
Multi-source localization in MEG using simulated annealing: Model order determination and parameter accuracy
}

\author{
Huang, M., ISupek, S., Aine, C. \\ Biophysics Group, Los Alamos National Laboratory, Los Alamos, NM, USA; \\ IDepartment of Physics, Faculty of Science, Zagreb, Croatia
}

\section{Introduction}

Empirical neuromagnetic studies have reported that multiple brain regions are active at single instants in time as well as across time intervals of interest [1]. Determining the number of active regions, however, required a systematic search across increasing model orders using a reduced chi-square measure of goodness-of-fit and multiple starting points within each model order assumed [2]. Such a time-intensive strategy was necessary since initial source modeling assumptions for a given instantaneous or spatio-temporal data set are not available a priori and the minimization algorithms typically used rely on either a gradient-based search or simplex, both of which are reported to be sensitive to the initial starting points [2,3]. Simulated annealing was recently proposed for noiseless biomagnetic data $[4,5]$ as an effective global minimizer. It does not require multiple starting points and with an appropriate cooling schedule, should converge to the global minimum. A modified cost function was also proposed to effectively deal with an unknown number of dipoles for noiseless, multi-source biomagnetic data [5].

Numerical simulation studies were conducted using simulated annealing as described in [6] to examine: 1) effects of a systematic increase in model order using both reduced chi-square [2] as a cost function as well as a modified cost function as proposed in [5]; and 2) effects of overmodeling on parameter estimation accuracy. Effects of different choices of weighting factors are also discussed. Simulated annealing was also applied to visually evoked neuromagnetic data and the effectiveness of both cost functions in determining the number of active regions was demonstrated.

\section{Method}

Instantaneous neuromagnetic field distributions were generated by 2 - and 3-dipole configurations embedded in a spherical conductor. Field values were calculated at 135 measuring points distributed over a portion of a spherical surface $(R=11.3 \mathrm{~cm})$ as recorded by first-order gradiometers. Normally distributed noise of zero mean and a standard deviation of $20 \mathrm{fT}$ was added. Simulated annealing was implemented as in [6]. The center of a spherical conductor was selected as the starting point in all cases. An iterative random search for dipole locations over the entire head volume was permitted. In order to approach the global minimum, the following annealing schedule was found as adequate: $T_{n+1}=(1-\varepsilon) T_{n}$, where $\varepsilon$ was 0.05 for noisy and 0.15 for noiseless cases examined, and $T_{n+1}$ and $T_{\mathbf{n}}$ are the temperature parameters for two consequent states. Best-fitting parameter estimates were obtained by minimizing: 1) chi-square $\left(\chi^{2}\right)$ as a cost function for every model order assumed [2]

$$
\chi^{2}=\sum_{i=1}^{N}\left(\frac{B_{i}-\widehat{B}_{i}\left(a_{j}\right)}{\sigma_{i}}\right)^{2}
$$

and 2) a modified cost function (MCF) as proposed by [5]

$$
\mathrm{MCF} \equiv \chi^{2}+w \sum_{p=1}^{P}\left|\widehat{B}_{p}\right|^{1 / 2}
$$

where $B_{i}$ are noisy simulated field values at every sensor location $i=1, \ldots N, \widehat{B}_{i}\left(a_{j}\right)$ are field values for an assumed model described by parameters $a_{j} ; \sigma_{i}$ are standard deviations of gaussian noise added assumed to be equal for every sensor; and $\widehat{\mathbf{B}_{\mathrm{p}}}$ are field values generated by $\mathrm{p}=1, \ldots \mathrm{P}$ dipoles assumed in the model, typically greater than the actual or expected number of sources; $w$ is a weighting factor. The second term in Eq.(2) is referred to as $E_{W}$. A range of weighting factors, used in the second term of the modified cost function, was examined. 


\section{Results}

Both cost functions permitted the identification of the actual number of sources for 2- and 3-dipole configurations under noiseless and noisy conditions. While $\chi^{2}$ required a search across model orders, a modified cost function allowed the proper identification of the actual number of sources when a model order greater than the actual number was assumed. However, different choices of weighting factors were necessary in order to assure: 1) adequate cost function values (at least for $\chi^{2}$ for which the probability distribution is known) were obtained; and 2) the moments of extra dipoles added were close to zero. Table I. represents actual source parameters (panel A) and bestfitting parameter estimates (panels B and C) for noisy 2-dipole data for both cost function values when the model orders assumed were 3 and 4 (both of which represented overmodeling). In cases where adequate weighting factors

Table I: Noisy 2-Dipole Data $(\mathrm{SD}=20 \mathrm{fT}$ )

\section{A. Actual Source Parameters}

\begin{tabular}{|c|c|c|c|c|}
\hline Dipole: & $\rho(\mathbf{c m})$ & $v\left({ }^{0}\right)$ & $\varphi\left({ }^{0}\right)$ & $P(\mathbf{n A m})$ \\
\hline 1 & 8.0 & 70.0 & 165.0 & 8.0 \\
\hline 2 & 8.0 & 70.0 & 195.0 & 8.0 \\
\hline
\end{tabular}

B1. Model order $=3 ; \mathbf{w}=\mathbf{0 . 0 2 5}$

$\begin{array}{llllllll}\text { Dipole: } & \rho(\mathrm{cm}) & v\left({ }^{(}\right) & \varphi\left({ }^{(}\right) & \text {P (nAm) } & \psi\left({ }^{(}\right) & \chi^{2} & \chi^{2}+\mathrm{E}_{\mathbf{w}} \\ \mathbf{1} & \mathbf{8 . 0 4 3} & \mathbf{7 0 . 9 6 6} & \mathbf{1 6 5 . 5 8 6} & \mathbf{7 . 8 5 4} & \mathbf{2 . 1 4 5} & 117.490 & 166.209 \\ \mathbf{2} & \mathbf{7 . 8 8 4} & \mathbf{7 0 . 7 0 1} & \mathbf{1 9 5 . 9 5 8} & \mathbf{8 . 5 8 4} & \mathbf{- 0 . 8 1 4} & & \\ 3 & 9.999 & 33.458 & 178.669 & 0.204 & 0.714 & & \end{array}$

B2. Model order $=3 ; \mathrm{w}=\mathbf{0 . 0 1}$

$\begin{array}{lll}1 & \mathbf{8 . 0 0 2} & \mathbf{7 0 . 3 4} \\ \mathbf{2} & \mathbf{7 . 9 5 8} & \mathbf{7 0 . 0 4} \\ 3 & 0.008 & 143.205\end{array}$

B3. Model order $=3 ; \mathbf{w}=\mathbf{0 . 0 2}$

$\begin{array}{lll}\mathbf{1} & \mathbf{8 . 0 4 0} & \mathbf{7 0 . 5 1 7} \\ \mathbf{2} & \mathbf{7 . 9 5 7} & \mathbf{7 0 . 5 1 2} \\ 3 & 0.000 & \mathbf{1 7 4 . 1 2 8}\end{array}$

165.987
196.196
309.478

$\begin{array}{ll}8.129 & 0.902 \\ 8.116 & -0.147 \\ 0.115 & 311.746\end{array}$

165.994
196.046
107.094

\subsection{1}

8.087

0.523

133.753

484.928

0.061

$-0.039$

219.199

B4. Model order $=3 ; \mathbf{w}=\mathbf{0 . 0 3}$

$\begin{array}{lll}1 & 6.416 & 67.896 \\ 2 & 0.009 & 95.623 \\ 3 & 0.000 & 28.366\end{array}$

\subsection{0 \\ 223.362}

310.637

$\begin{array}{ll}19.998 & 0.000 \\ 0.000 & 333.031 \\ 1.371 & 21.174\end{array}$

397.362

729.431

C1. Model order $=4, \mathbf{w}=\mathbf{0 . 1 0}$

\begin{tabular}{lll}
$\mathbf{1}$ & $\mathbf{8 . 0 5 2}$ & $\mathbf{7 1 . 0 7 9}$ \\
$\mathbf{2}$ & $\mathbf{7 . 8 9 4}$ & $\mathbf{7 1 . 2 6 4}$ \\
3 & 0.000 & $\mathbf{1 3 7 . 9 7 8}$ \\
4 & 0.000 & $\mathbf{1 3 8 . 8 8 7}$ \\
\multicolumn{4}{c}{ C2. Model order $=4}$, & $\mathbf{w}=\mathbf{0 . 0 2}$ \\
$\mathbf{1}$ & $\mathbf{8 . 0 9 9}$ & $\mathbf{7 1 . 4 6 2}$ \\
$\mathbf{2}$ & $\mathbf{7 . 8 8 7}$ & $\mathbf{7 1 . 5 0 8}$ \\
3 & 0.721 & 116.628 \\
4 & 1.356 & 117.025
\end{tabular}

165.657

7.837

$-2.095$

131.266

307.815

$\begin{array}{lll}195.789 & 8.551 & 2.815\end{array}$

$\begin{array}{lll}169.243 & 0.155 & 152.699\end{array}$

223.289

0.6475

308.489

165.729

7.548

195.585

8.516

$-3.374$

134.262

483.349

212.920

0.000

4.240

79.465

116.144

0.000

167.918 
were selected, accurate parameter estimation of the actual sources was possible and the addition of extra dipoles resulted only in minor effects on parameter estimation accuracy. The moments of the extra dipoles were very close to zero (panels B2, B3, C1, C2) and their estimated locations were close to the center of the spherical conductor. However, selecting too large of a weighting factor (panel B4), strongly affected parameter estimates of all sources and resulted in too improbable $\chi^{2}$ values indicating inadequacy of the model assumed. In this case, it was assumed that the particular choice of the weighting factor was inadequate, since overmodeling itself al ways lead to adequate $\chi^{2}$ values when an optimal solution is reached. The optimal weighting factors for 3-dipole noisy data (not shown) were different than for the 2-dipole case (Table I.) (e.g., $w=0.075$ for an assumed model order of 4 and $w=0.065$ for an assumed model order of 5, respectively). While Haneishi and colleagues [5] suggested a weighing factor of about 0.5 for noiseless data, we found empirically that the ratio of $2: 3$ between first and second term in Eq.(2), resulted in an adequate choice of weighting factors for noisy simulated conditions.

Table II. shows best-fitting parameter estimates and cost-function values when different multi-dipole models were applied to visual neuromagnetic data evoked by a small Target stimulus placed $4^{\circ}$ below the horizontal meridian and $3^{\circ}$ to the right of the vertical meridian [8]. A 2-dipole model was identified in this case as an adequate model for an instantaneous field distribution at an early latency of $110 \mathrm{~ms}$ poststimulus by both cost functions. The identified brain regions were in agreement with solutions obtained by spatio-temporal modeling of the early evoked activity from $80-120 \mathrm{~ms}$. Reduced chi-square values, rather than $\chi^{2}$, are presented in panel A.

Table II. Visually evoked neuromagnetic data $(\mathrm{SD}=25.44 \mathrm{fT})$

\section{A. Reduced chi-square - Increasing model order}

$\begin{array}{llllllll}\text { Dipole: } & \mathbf{x}(\mathbf{c m}) & \mathbf{y}(\mathbf{c m}) & \mathbf{z}(\mathbf{c m}) & \mathbf{P}(\mathbf{n A m}) & \psi(\mathbf{0}) & \chi \mathbf{r}^{2} & \mathrm{Q} \\ \mathbf{1} & -4.93 & 1.23 & 7.03 & 6.37 & 173.93 & 1.479 & 0.007 \\ & & & & & & & \\ \mathbf{1} & \mathbf{- 6 . 5 6} & \mathbf{0 . 5 5} & \mathbf{5 . 0 3} & \mathbf{5 . 2 2} & \mathbf{2 2 0 . 9 7} & 0.967 & 0.550 \\ \mathbf{2} & \mathbf{- 4 . 3 9} & \mathbf{3 . 0 9} & \mathbf{5 . 2 3} & \mathbf{8 . 4 8} & \mathbf{1 1 5 . 5 2} & & \\ & & & & & & & \\ \mathbf{1} & -7.20 & 0.02 & 4.96 & 2.97 & 219.32 & 0.867 & 0.749 \\ 2 & -4.91 & 2.44 & 5.57 & 7.18 & 140.05 & & \\ 3 . & -7.55 & 4.86 & 4.02 & 0.20 & 17.71 & & \end{array}$

B. Modified cost function - Overmodeling: Model order $=4 ; \mathrm{w}=0.046$

$\begin{array}{llllllll}\text { Dipole: } & \mathbf{x}(\mathbf{c m}) & \mathbf{y}(\mathbf{c m}) & \mathbf{z}(\mathbf{c m}) & \mathbf{P}(\mathbf{n A m}) & \psi\left(\mathbf{(}^{\circ}\right) & \chi^{2} & \chi^{2}+\mathbf{E}_{\mathbf{w}} \\ \mathbf{1} & \mathbf{- 6 . 7 4} & \mathbf{0 . 7 2} & \mathbf{5 . 1 1} & \mathbf{3 . 8 9} & \mathbf{2 1 6 . 5 4} & 54.36 & \mathbf{8 8 . 0 3} \\ \mathbf{2} & \mathbf{- 4 . 6 8} & \mathbf{3 . 6 0} & \mathbf{5 . 1 6} & \mathbf{5 . 2 1} & \mathbf{1 1 7 . 9 9} & & \\ \mathbf{3} & -7.59 & -4.26 & 8.33 & 0.08 & 54.51 & & \\ 4 & 0.00 & 0.00 & 4.01 & 0.02 & 298.93 & & \end{array}$

\section{Discussion}

Both cost functions allowed for the identification of the actual number of sources in simulated cases (Table I) and the same adequate model order in empirical data (Table II). Best-fitting source parameters were either accurate estimates of the actual source parameters or represented physiologically plausible solutions which were similar to those obtained previously using other methods [7]. While $\chi^{2}$ required a systematic increase in model order as demonstrated earlier [1], the use of a modified cost function allowed for more efficient model order determination 
while preserving the accuracy of parameter estimation. In essence, if one applies a model order which represents overmodeling, the correct number of dipoles can still be obtained since the "extra dipoles" would either have close to zero moments and they would be placed near the center of the head. Consequently, these "extra dipoles" are easy to identify. However, while a systematic search over different model orders might be avoided by using a modified cost function as proposed by [5], consideration of different weighting factors was necessary since both previous [5] and present simulations indicate that optimal values should be found empirically. Therefore, a search over model orders or over a range of weighting factors seems unavoidable. Since we found the optimal choice of the weighting factor to be related to the actual source configuration, which is unknown in empirical cases, it turns out that a search over model orders is likely to be more efficient. Previous simulations [5], as well as the present results, demonstrate the promise of simulated annealing as an efficient minimization approach for neuromagnetic applications. We are currently implementing simulated annealing for spatio-temporal analysis of neuromagnetic data [8] with $\chi^{2}$ as a cost function of choice.

\section{References:}

[1] Aine, C.J., Supek, S., and George, J.S. Temporal dynamics of visual-evoked neuromagnetic sources: Effects of stimulus parameters and selective attention, Intern. J. Neuroscience, 1995, 80: 79-104.

[2] Supek, S., and Aine, C.J. Simulation studies of multiple dipole neuromagnetic source localization: Model order and limits of source resolution, IEEE Trans. Biomed. Eng., 1993, BME-40: 529-540.

[3] Press, W.H., Teukolsky, S.A., Vettering, W.T., and Flannery, B.P. Numerical Recipes in C, Cambridge University Press, 1992

[4] Sekihara, K., Haneishi, H., and Ohyama, N. Details of simulated annealing algorithm to estimate parameters of multiple current dipoles using biomagnetic data, IEEE Trans. Med. Eng., 1992, BME-11: 293-299

[5] Haneishi, H., Ohyama, N, Sekihara, K., and Honda, T. Multiple current dipole estimation using simulated annealing, IEEE Trans. Med. Eng., 1994, BME-41: 1004-1009

[6] Corana, A., Marchesi, M., Martini, C., and Ridella, S., Minimizing multimodal functions of continuous variables with the "simulated annealing" algorithm, ACM Trans. Mathem. Software, 1987, 13: 262-280

[7] Aine, C.J., Supek, S., George, J.S., Ranken, D., Lewine, J., Sanders, J., Best, E., Tiee, W., Flynn, E.R., and Wood, C.C. Retinotopic organization of human visual cortex: Departures from the classical model (in press, Cerebral Cortex, 1996)

[8] Huang, M., Supek, S., and Aine, C., Application of simulated annealing for multi-source spatio-temporal localization in MEG: Simulation and empirical study, 2nd International Conference on Functional Mapping of the Human Brain, June 17-21, 1996.

\section{Acknowledgement}

This research was supported in part by a grant from NEI (EY08610) and by the United States Department of Energy, contract W-7405-ENG-36.

\section{DISCLAIMER}

This report was prepared as an account of work sponsored by an agency of the United States Government. Neither the United States Government nor any agency thereof, nor any of their employees, makes any warranty, express or implied, or assumes any legal liability or responsibility for the accuracy, completeness, or usefulness of any information, apparatus, product, or process disclosed, or represents that its use would not infringe privately owned rights. Reference herein to any specific commercial product, process, or service by trade name, trademark, manufacturer, or otherwise does not necessarily constitute or imply its endorsement, recommendation, or favoring by the United States Government or any agency thereof. The views and opinions of authors expressed herein do not necessarily state or reflect those of the United States Government or any agency thereof. 